\title{
Recent Beauty Results from CDF and the Run-II Upgrades
}

\author{
B. Todd Huffman \\ For the CDF Collaboration \\ University of Pittsburgh \\ Pittsburgh, Pennsylvania 15260
}

Fermi National Accelerator Laboratory

P.O. Box 500, Batavia, Illinois 60510

September 1996

Published Proceedings of Beauty 96, Rome, Italy, June 17-21, 1996. 


\section{Disclaimer}

This report was prepared as an account of work sponsored by an agency of the United States Government. Neither the United States Government nor any agency thereof, nor any of their employees, makes any warranty, express or implied, or assumes any legal liability or responsibility for the accuracy, completeness or usefulness of any information, apparatus, product or process disclosed, or represents that its use would not infringe privately owned rights. Reference herein to any specific commercial product, process or service by trade name, trademark, manufacturer or otherwise, does not necessarily constitute or imply its endorsement, recommendation or favoring by the United States Government or any agency thereof. The views and opinions of authors expressed herein do not necessarily state or reflect those of the United States Government or any agency thereof.

\section{Distribution}

Approved for public release: further dissemination unlimited. 
FERMILAB-CONF-96/312-E

CDF/PUB/BOTTOM/PUBLIC/3858

\title{
Recent Beauty Results from CDF and the Run-II Upgrades
}

\author{
B.Todd Huffman ${ }^{1}$ \\ for the CDF Collaboration \\ University of Pittsburgh \\ Pittsburgh, PA 15260
}

\begin{abstract}
This paper reports on the most recent results from the Collider Detector Facility (CDF) from $110 \mathrm{pb}^{-1}$ of data taken at the Fermilab Tevatron from 1992 to 1995 . Improved measurements of $B$ meson spectroscopy, lifetimes, and searches for new particle states are presented. Evidence for the effectiveness of same side tagging techniques in the context of mixing measurements is shown with applicability for CP violation studies in Run-II explained. The planned upgrades of importance to $b$ physics for Run-II are briefly detailed with an emphasis on the expected physics reach in Run-II by CDF.
\end{abstract}

\footnotetext{
${ }^{1}$ nimi@inal.gor

Published Proceedings Beauty 96, Rome, Italy, June 17-21, 1996
} 


\section{Introduction}

Bottom mesons are detectable in high energy hadron colliders by the fact that they are long-lived with a lifetime of about $1.5 \mathrm{ps}$. At the Tevatron this lifetime imparts an average displacement of $600 \mu \mathrm{m}$ to the $B$ meson before it decays. By surrounding the interaction region with a silicon detector capable of finding the displaced vertex and a tracking spectrometer, CDF has shown that it is possible to compete effectively with the more traditional $e^{+} e^{-}$colliders in certain areas of the study of bottom quarks and mesons.

Despite possessing the largest production rate of $B$ mesons in the world ( $\simeq 30 \mu \mathrm{b}$ for $|y|<1.0$ ), the background rate from normal $p \bar{p}$ collisions limits the decays studied to those involving $J / \psi$ and lepton final states. Triggering on electrons and muons helps remove the QCD background. Even so, interesting results on $B$ cross sections, $B, B_{a}$, and $\Lambda_{b}$ lifetimes, mixing, new particle searches, ratios of branching ratios, final state polarizations, and tagging methods have been reported by CDF in the last year.

Section 2 of this report focuses on the most recent results from CDF with data from Run-I of the Fermilab Tevatron. Section 2 will also detail an analysis on the technique of same side tagging of $B$ mesons in the context of a mixing measurement but with implications studying $\mathrm{CP}$ violation in the $B$ sector in Run-II. Section 3 will briefly describe the expected beam parameters for Run-II and the planned detector upgrades to CDF. Finally section 4 will project the expected physics reach of CDF in Run-II for measuring CP violation and other studies of the bottom meson.

\section{Recent CDF Results}

The data used in these analyses were collected with the Collider Detector at Fermilab (CDF) during the 1992-1995 run, and correspond to an integrated luminosity of $110 \mathrm{pb}^{-1}$ from $\bar{p} p$ collisions at $\sqrt{s}=1.8 \mathrm{TeV}$. The CDF detector is described in detail elsewhere [1]. We describe the components of the detector that are important for these analyses. The silicon vertex detector (SVX) and the central tracking chamber (CTC) provide spatial measurements in the $r-\varphi$ plane 2 ], giving an average track impact parameter resolution of $\sim\left(16+30 / P_{T}\right) \mu \mathrm{m}$, where $P_{T}$ is the track transverse momentum in units of $\mathrm{GeV} / c$. The $P_{T}$ resolution of the CTC combined with the SVX is $\delta\left(P_{T}\right) / P_{T}=\left((0.0066)^{2}+\left(0.0009 P_{T}\right)^{2}\right)^{1 / 2}$ [3]. Two muon subsystems in the central region were used, which together provide coverage 
in the pseudo-rapidity interval $|\eta|<1.0$, where pseudo-rapidity is defined as $\eta=-\ln [\tan (\theta / 2)]$.

In order to detect the decays of the $B$ mesons CDF uses a multi-layer trigger system that triggers on single leptons, muon pairs, or on electron-muon events. Level 1 triggers on hits in the muon chambers or electromagnetic calorimeter showers. Level 2 matches tracks to these hits and requires multiple hits in the case of dileption and $e-\mu$ triggers. Both levels 1 and 2 are hardware triggers. Level 3 is in software and improves the resolution and cuts of the triggers if necessary.

\subsection{Hadronic b Production}

Initial measurements of the $J / \psi$ and $\psi(2 S)$ cross section from $B$ decays seemed to indicate larger production rates than predicted. [4] Recent data has subsequently shown that most $J / \psi$ and $\psi(2 S)$ production is prompt. Shown in Figure 1 is the fraction of $J / \psi$ candidates originating from $B$ meson decays. When this fraction is removed and a Monte Carlo of the detector used to correct for the acceptance as a function of transverse momentum $\left(P_{T}\right)$, the total cross sections for the production of prompt $J / \psi$ and $\psi(2 S)$ are obtained. Figure 2 shows the differential cross sections as a function of $P_{T}(\psi, \psi(2 S))$. The theory curve is a color octet model [5] that was normalized to an earlier subset of the data shown here. This model also predicts that the charmonium states should be polarized at high momentum relative to their masses. Work is in progress to determine if this is observed.

By looking at the data which exhibits a long lifetime the cross section of $J / \psi$ and $\psi(2 S)$ states from $B$ meson decays can be obtained. Figure 3 shows the resulting differential cross sections. The two lines are calculations of the two cross sections based on NLO QCD [6], MRSD0 structure functions [7], Peterson fragmentation [8], and the CLEO decay module for the $B \rightarrow \psi+X$ process. As has been reported previously, the cross section from $B$ decays is above the prediction; however, it is possible to alter the QCD energy scale, fragmentation $\epsilon$, and the $b$ quark pole mass to get within one standard deviation of the observed value. We are beginning to observe that the shape of theory does not agree with observed $B$ spectrum for $J / \psi P_{T}$ smaller than $\simeq 7.0 \mathrm{GeV} / \mathrm{c}$. The $\psi(2 S)$ spectrum is still consistent with the predicted shape within statistics.

Because there is additional uncertainty in the $B$ meson spectrum introduced by the decay to the $\psi$, measuring the production cross section in exclusive final states is also important. Shown in Figure 4 is the $B$ meson 
cross section where the meson was detected from the decays $B \rightarrow J / \dot{\psi}+\dot{K}$ where both $K^{ \pm}$and $K^{* 0}, \bar{K}^{* 0}$ are included. The charged mode contains $73 \mathrm{pb}^{-1}$ of data while the neutral mode contains $19 \mathrm{pb}^{-1}$. The difference in normalization between predicted and observed values is preserved while the shape agrees well with the exception of the lowest momentum bin.

The high production rate of $J / \psi B$ mesons at the Tevation allows precision measurements of the ratio of branching ratios between $B \rightarrow J / \psi+K$ and $B \rightarrow \psi(2 S)+K$ in both charged and neutral modes. $\frac{\mathrm{BR}\left(\psi(2 S)+K^{+}\right)}{\mathrm{BR}\left(J / \psi+K^{+}\right)}=$ $0.67 \pm 0.09 \pm 0.10$ while $\frac{\mathrm{BR}\left(\psi(2 S)+K^{* 0}\right)}{\mathrm{BR}\left(J / \psi+K^{\circ 0}\right)}=0.57 \pm 0.13 \pm 0.07$

\subsection{New Particle Searches}

Hadron colliders produce all types of the $B$ hadrons. Some of these states may prove useful in the study of QCD final state interactions and in mixing or $\mathrm{CP}$ violation analyses.

Earlier this decade, the $\Lambda_{b}$ baryon was claimed to have been seen at UA1 [9] and has subsequently been seen at LEP.[10] CDF observes $13.7 \pm 5.3$ $\Lambda_{b} \rightarrow J / \psi+p+\pi$ candidates compared to $87.9 \pm 11.2 B_{d} \rightarrow J / \psi+K_{d}^{0}$ decays. Factoring out the expected production rate and $B_{d}$ branching ratio, $\operatorname{BR}\left(\Lambda_{b} \rightarrow J / \psi+\Lambda\right)=4.3 \pm 2.1 \pm 0.8 \times 10^{-4}$ is obtained; a factor of 30 smaller than the previous hadronic result and consistent with the rates seen at LEP.

The bottom-charmed meson $\left(B_{c}\right)$ could be produced at the Tevatron with a rate as high as $10^{-3}$ times the production of the other $B$ hadrons. [11] Though the spectroscopy is predicted with high precision[12], the initial and final state interactions of this weakly decaying heavy bound state make predictions of its lifetime uncertain.[13].

CDF is currently searching in two channels for this particle. The decay $B_{c} \rightarrow J / \psi+l+X$ is not fully reconstructed and has backgrounds that are currently under study. Reported here is the search in the exclusive decay $B_{c} \rightarrow J / \psi+\pi$ with the entire $110 \mathrm{pb}^{-1}$ of data. Figure 5 shows the limit as a function of the assumed lifetime in the mass region from 6.1 to $6.4 \mathrm{GeV} / \mathrm{c}^{-2}$ with respect to the production of the kinematically similar decay of $B_{u} \rightarrow J / \psi+K$. CDF has also observed the Cabbibo suppressed decay $B_{u} \rightarrow J / \psi+\pi$ and has obtained $\frac{\mathrm{BR}(J / \psi+\pi)}{\operatorname{BR}(J / \psi+K)}=5.0_{-1.7}^{+1.9} \pm 0.1 \%$. 


\subsection{Lifetimes}

Studying the lifetimes of the charged and neutral $B$ mesons and the $\Lambda_{b}$ probes final state interactions and tests the prediction of the factorization hypothesis that all $B$ hadrons will have nearly the same lifetime. CDF has recently updated results with the full data set in the inclusive decay of the $B$, to $D$, lepton, inclusive $J / \psi$ 's and in the fully reconstructed modes of the $B$ meson. Figure 6 shows the psuedo-ct distribution of the $J / \psi$ sample. Using the long-lived component of this plot we determine that $\tau_{\text {binc }}=1.524 \pm 0.015_{-0.026}^{+0.035} \mathrm{ps}$ where the first uncertainty is statistical and the second systematic. Shown in Table 1 are the remaining results on lifetimes from CDF with the LEP averages also shown for comparison purposes. The LEP results shown are those presented at this conference.[14]

\subsection{Same Side Tagging of $B$ Mesons}

Studies are continuing at $C D F$ to improve methods of identifying the flavor of the $B$ meson when it is produced. This is important for two types of measurements; mixing and $\mathrm{CP}$ violation of the $B^{0}$ or $B_{a}^{0}$ mesons.

In a mixing or $\mathrm{CP}$ violation experiment there are three parts to the analysis. The particle type must be identified at decay by the decay products, the decay length must be measured, and the meson type when it was produced identified.

The meson type at decay time is identified by finding a lepton $+D^{0}\left(D^{+}, D^{*}\right)$ state. The sign of the lepton then determines whether the meson was a $B$ or $\bar{B}$ and the charge of the $D$ determines whether the $B$ was neutral or charged.

The lepton and the $D$ meson daughter tracks are required to be in the SVX. The tracks forming the $D$ meson are vertex constrained and the resulting parent trajectory projected back to form a vertex with the lepton thus identifying the location of the $B$ meson decay.

At hadron colliders the most difficult part of this analysis is the identification of the $B$ meson flavor at production. One method of identification suggested in the literature[15] was to identify the soft charged pions that should be shed during $B$ fragmentation to maintain color neutrality or the pion daughters of the decays of higher resonance states (like $B^{* *}$ ). In both cases the charge of the associated pion is correlated the same way with the flavor of the parent meson.

We use the track with the minimum $p_{T}^{\text {rel }}$ other than the lepton $+D$ tracks 
within $R=\sqrt{\eta^{2}+\varphi^{2}} \leq 0.7$ as the candidate pion track thus tagging the flavor of the initial meson. The average number of tracks in the cone per event is 3.2 not including the lepton $-D$ tracks. With this identification method one can plot the time-dependent asymmetry in the number of "right" vs. "wrong" sign tags.

$$
A(t) \equiv \frac{N_{R S}(t)-N_{w \vartheta}(t)}{N_{R S}(t)+N_{w_{\vartheta}}(t)}
$$

Equation 1 should have no time dependence for charged $B$ 's, but it will take the form $D_{0} \cos \left(x_{d} t / \tau\right)$ for neutral $B$ 's because of mixing. $D_{0}$ is defined as the "dilution" of the sample and will be $\left|D_{0}\right| \leq 1.0 . N_{R S}(t)$ and $N_{w}(t)$ are simply the number of events as a function of $c \tau$ where the flavor tagging track matches or doesn't match the expected flavor of the lepton $+D$ system. The analysis was done for both charged and neutral $B$ meson final states as a comparison and as a measure of the quantity $D_{0}$ called the dilution. This quantity would equal 1.0 for charged $B$ mesons and would produce a time dependent amplitude of 1.0 in the neutral case if the tagging method were perfect.

\subsubsection{Corrections to the Dilution $\left(D_{0}\right)$ and Final Result}

There are several sources of contamination that come in from other decays of $B$ mesons. Among examples of these are the decays $B^{0} \rightarrow \nu l^{+} D^{* *-}$ and $B^{0} \rightarrow \nu l^{+} D^{*-}$. The first decay sheds a pion in the decay chain of the $D^{* *}$ that can be missidentified as a valid tag while the second decay would fake a $B^{+} \rightarrow \nu l^{+} \bar{D}^{0}$ if the soft pion from the $D^{*}$ is missed. By comparing the number of pion tags that are consistent with the $B$ vertex as opposed to the primary vertex in data one can measure the effect of these backgrounds. Corrections are also applied to account for the SVX resolution.

After the background subtractions and corrections are applied the resulting asymmetry vs. decay length is plotted in Figure 7 for $B^{+}$and $B_{0}$ respectively. There is clear evidence for the mixing of the neutral mesons and no indication the charged mesons mix. The values obtained are $x_{d}=0.67 \pm 0.09$, $D_{+}=0.28 \pm 0.04$, and $D_{0}=0.22 \pm 0.03$. Though the combined LEP measurement of $x_{d}$ is to higher precision, the point of this observation is to show the effectiveness of same-side tagging. Ultimately same side tagging will be combined with other CDF tagging methods for a more accurate measure of $B-\bar{B}$ mixing. 


\section{CDF Upgrade Plans for Run-II}

The last run of the Tevatron ended in 1996. Fermilab is constructing a new main injector that will enable closer spacing of bunches and will facilitate higher luminosity. The next run of the Tevatron with the main injector is scheduled for 1999. Run-II is the designation for this colliding beam run.

There are several challenges that must be met by CDF in order to effectively take data during Run-II. The projected maximum luminosity is expected to approach $2 \times 10^{32} \mathrm{~cm}^{-2} \mathrm{~s}^{-1}$. The run will begin with 36 proton and antiproton bunches $396 \mathrm{~ns}$ apart and could go to 99 bunches with $132 \mathrm{~ns}$ spacing by the end of the run. The integrated radiation $2.5 \mathrm{~cm}$ from the beam line will reach $1 \mathrm{Mrad}$ and it is expected that $2 \mathrm{fb}^{-1}$ of data can be delivered during the course of the run. Constructing a detector capable of operating under these conditions requires the replacement and changing of several key systems in the Collider Detector.

This high luminosity running presents beauty physics with another opportunity to take advantage of the high production rate at the Tevatron in Run-II, and the CDF collaboration is focusing resources on detectors and data acquisition that could unlock nearly all decays of the $B$ meson rather than the traditional $J / \psi$ and lepton triggers. Specifically a silicon detector (the SVX-II) will send data to a trigger that searches for tracks with high impact parameter (the SVT). This trigger and silicon detector will allow CDF to trigger on non-leptonic tracks from $B$ meson decay for the first time. There are upgrade plans for other systems in the Collider Detector but this paper will focus on the upgrades that will effect the study of $B$ mesons most dramatically and on the physics capabilities thus gained for Run-II.

\subsection{The Trackers}

\subsubsection{Central Open-cell Tracker (COT)}

The current Central Tracking Chamber (CTC) will require replacement. The CTC was in use for the 1987 running of the Collider Detector when the instantaneous luminosity of the Tevatron was two orders of magnitude smaller than in Run-I. As the luminosity has increased the inner layers of the CTC have been forced to contend with increased occupancy and space charge in the drift volume. To counteract those effects the gain of the chamber was reduced for Run-I and the sensitivity of the preamplifiers increased to compensate. For the yet higher luminosity of Run-II, the number of 
interactions per crossing is controlled by increasing the number of bunches thus decreasing the crossing time from $3500 \mathrm{~ns}$ to $396 \mathrm{~ns}$ (and eventually to $132 \mathrm{~ns}$ ). However, the CTC, with 700 ns maximum drift time, cannot take full advantage of the increased number of bunches.

As a result a new tracking chamber will be needed for Run-II that would be capable of resolving individual beam crossings even if the time between bunches drops to $132 \mathrm{~ns}$. This proposed tracker is called the central open-cell tracker (COT).

The COT will consist of 8 superlayers of cells. One-half of these cells will have sense wire planes on $a 3^{\circ}$ angle with respect to the beam for stereo $z$ resolution. Each cell has 12 sense wires, uses a fast gas, and a small drift region so that the maximum drift time is expected to be $100 \mathrm{~ns}$ when the crossing time is $132 \mathrm{~ns}$. The COT has approximately twice the number of wires as the current tracker but the same number of radiation lengths of material. Table 2 contains a summary of the properties of the COT and the CTC for comparison. Apart from being more radiation resistant and capable of delivering information faster than the CTC, the COT is expected to have the same track resolution as the CTC.[16]

\subsubsection{The Silicon Vertex Detector (SVX-II)}

The CDF collaboration has had the benefit of silicon vertex detectors in the form of the SVX and SVX' for all of Run-I. These devices were key to giving the collaboration enough confidence to present preliminary results on the discovery of the top quark in 1994.[17] They are also the primary reason that CDF can compete at all with the experiments at CLEO and LEP in the realm of $b$ physics.

The worth of this type of detector is such that there is now little question silicon detectors should be placed at the heart of any colliding beam experiment. Hence the SVX-II will replace the current SVX' near the luminous region. The SVX-II detector will supply data to the level 2 trigger. This as well as the increased radiation fluence drive the design parameters of the SVX-II.

The physical design of the detector will cover over $90 \%$ of the luminous region, and SVX-II will have double sided detectors that will provide full 3-d vertexing. One detector unit is termed a "barrel" and each barrel contains 12 radial slices in $\phi$ with 5 layers each. A total of 3 barrels will make up SVX-II. Table 3 lists the main points of the SVX-II and compares them to the current SVX' detector. 
The SVX-II silicon detector will have 5 layers of silicon radially from the beam. Layers 0,1 , and 3 will have $\phi$ and $z$ strips at $90^{\circ}$ with respect to each other whereas layers 2 and 4 will have stereo strips at an angle of $1.2^{\circ}$. The first layer of silicon encountered from the beam will be located $2.55 \mathrm{~cm}$ from the beam. There are a factor of ten more channels in this detector compared to the SVX'. In order to accommodate this and to supply the trigger the readout speed is a factor of 25 times faster and has more parallel links so the data rate from the entire detector is $153 \mathrm{Gbits} / \mathrm{s}$ when readout begins. It is expected that the single track resolution should be similar to the SVX in the $r-\phi$ plane $(\simeq 8 \mu \mathrm{m})$ and equal to the strip pitch divided by $\sqrt{12}$ for normal incident tracks but better resolution $(\simeq 25 \mu \mathrm{m})$ when more than one strip is hit in the $r-z$ plane.[16]

\subsection{Data Acquisition}

\subsubsection{Deadtimeless Operation}

The entire data aquisition system for the Collider Detector will be upgraded to allow for a pipelined and buffered readout. The pipeline is determined by the length of time required to make a Level 1 trigger decision and return a Level 1 accept to the individual subsystems (like the SVX or the calorimeter). Shown in Figure 8 is a block diagram of the "deadtimeless" data acquisition system. Data is taken synchronously with the beam crossing and stored in a pipeline. When the event comes to the end of the pipeline it is gated (or not) by the presence of a Level 1 accept and a buffer number. This decision involves the electro-magnetic calorimeter, the central tracker, or the muon system. The level 1 decision and return time is estimated to be $5 \mu \mathrm{s}$.

If an event has been accepted it is stored in the buffer while the level 2 decision is made. The full level 2 time varies but is expected to average $20 \mu \mathrm{s}$. The level 2 trigger combines the track stubs in the SVX with the CTC and looks for high impact parameter tracks. It also makes matches to muons and electrons allowing for more discriminating power in the trigger.

When a level 2 accept or reject is received the buffer in question is either flushed or held while the event is scanned into the level 3 processing farm. The event rate into level 3 is expected to be $1 \mathrm{kHz}$. Until this point the trigger system was implemented in hardware or programmable gate arrays. Level 3 is a software trigger that pairs the rate down to $100 \mathrm{hz}$ for writing to tape (the final media will not be decided until closer to the run). All of 
this is controlled by a central processor called the Trigger Supervisor.

From the point of $B$ physics one of the key triggers in the level 2 trigger is the SVT or Silicon Vertex Trigger. This device accepts data from the SVX-II detector and the track list from the level 1 CTC trigger. First it finds the clusters and centroids using a hit finder. The locations of the hits found is sent to the associative memory which uses pre-designated track roads of $250 \mu \mathrm{m}$ to find SVX tracks. The roads are sent to a hit buffer where information from the CTC trigger at level 1 is also collected. This is where a road is associated with a particular CTC track. The combination is sent to a group of track fitter modules where the track finding process is refined up to the full spacial resolution of the SVX-II. Groups of track fitters are assembled into processor farms that are numerous enough that each farm should be able to work with only one road. The processor farm will find all the tracks in that road and return the impact parameter information. A pass/fail is then sent to the trigger supervisor. Figure 9 shows the path of data as it arrives from the SVX and the CTC through the processors described in this paragraph.

The SVT is segmented in $\phi$ in 12 sections as is the SVX. Any track that crosses wedge boundaries will not be found by the SVT unless a sufficient number of hits exist in one wedge. The SVT hit finders are independently pipelined so that information can be streaming into the hit finders while previous events are being processed further along. [16]

\section{Physics Prospects in Run-II}

With the addition of double sided silicon detector, pipelined and deadtimeless data acquisition, and the ability to trigger at level 2 on tracks with high impact parameter, CDF is well positioned to contribute to the next generation of measurements that can be made in the $B$ sector. Specifically finding the $B_{c}$ meson and measuring its lifetime may also be possible as well as searches for rare $B$ decays. Depending on $x_{s}$, it might also be possible to measure the oscillations of the $B$, meson. Additionally $\mathrm{CP}$ violation in $B$ decay should be observable at the Tevation.

\subsection{CP Violation Reach in Run-II}

When both the $B$ and $\bar{B}$ meson decay to the same CP eigenstate it is possible that the decay amplitudes will interfere. If the relative phase between the two amplitudes is not zero this effect will appear as an asymmetry in the 
decay rate of the $B-\bar{B}$ to that eigenstate. This will happen whether or not the $B$ meson is charged; however, if the neutral $B$ meson is studied that asymmetry will be time dependant and will take the following form:

$$
A(t)=\frac{\Gamma\left(B^{0}\right)-\Gamma\left(\bar{B}^{0}\right)}{\Gamma\left(B^{0}\right)+\Gamma(\bar{B})^{0}}=\frac{\left(1-|\lambda|^{2}\right) \cos x t-2 \operatorname{Im}(\lambda) \sin x t}{1+|\lambda|^{2}}
$$

Tree level diagrams are expected to dominate the decay amplitudes for the decay $B^{0} \rightarrow J / \psi+K_{0}^{0}$ which would imply for this case that $|\lambda| \simeq 1.0$ reducing equation 2 to a pure sine function. In this case the analysis proceeds much like the mixing analysis presented earlier in this proceeding. In fact, the problem of identifying the flavor of the $B$ meson at production is identical and causes a degradation (called the "dilution" $D=\left(N_{R}-N_{W}\right) /\left(N_{R}+N_{W}\right)$ where $N_{R}$ and $N_{W}$ are the number of right and wrong tags respectively.) when the initial flavor is not consistently tagged in the time dependent asymmetry. The effect of a diluted $A(t)$ is to require higher statistics to achieve the same accuracy on a measurement of the CP angle. The relationship between the $\mathrm{CP}$ asymetry uncertainty and the dilution is $\delta A^{2} \simeq 1 / N \epsilon D^{2} \frac{S+B}{S}$. Where $D$ is the dilution, $N$ is the total number of decays to the specific CP eigenstate, $S$ and $B$ are 'signal' and 'background' respectively, and $\epsilon$ is the $B$ flavor tagging efficiency. One can see that in addition to the statistics collected, the quantity $\epsilon D^{2}$ provides a figure of merit for determining what physics reach can be expected for Run-II.

\subsection{1 $\sin 2 \beta$}

In case of measuring $\sin 2 \beta$, the most practical method is to find and tag the decay $B^{0} \rightarrow J / \psi+K^{0}$. Shown in Figure 10 is a plot of the total number of events of this type collected in $110 \mathrm{pb}^{-1}$ of data. Several changes to the $J / \psi$ trigger are planned for Run-II and should increase the number of $J / \psi$ 's per $\mathrm{pb}^{-1}$ collected. The muon trigger acceptance will be improved and the muon momentum threshold on the trigger will be dropped from $2.0 \mathrm{GeV} / \mathrm{c}$ to $1.5 \mathrm{GeV} / \mathrm{c}$. This change should double the $J / \psi$ rate over Run-I implying approximately $10,000 \mathrm{~J} / \psi+K_{s}^{0}$ events by the end of Run-II.

Table 4 shows a list of current tagging methods that will be used in Run-II for mixing and CP violation measurements. Beside the list of current tagging methods being studied is a column with the measured values of $\epsilon D^{2}$ from the Run-I data and the expected values for Run-II. The last column is the primary upgrade responsible for the increase. Using upgraded values for $\epsilon D^{2}$ one would expect greater sensitivity to $\sin 2 \beta$. Additionally, an 
electron $J / \psi$ trigger will be added which should further improve statistics. Space is being provided for a time-of-flight system as well.[16] We estimate based on these improvements that CDF should be able to measure $\delta \sin 2 \beta$ to $0.08-0.13$. Since the expected value of $\sin .2 \beta=0.65 \pm 0.12$, a reasonable measurement should be possible.[18]

\subsection{2 $\sin 2 \alpha$}

Independently measuring the angles of the unitarity triangle overconstrains it and provides a test of the Standard Model. Consequently, one would like to try to measure $\sin 2 \alpha$ as well. CDF's "deadtimeless" DAQ system and the addition of the SVT will allow a high-rate, hadronic $B$ meson trigger at the Tevatron. This trigger could be used to look for CP violation in the decay $B^{0} \rightarrow \pi^{+} \pi^{-}$.

The level 1 trigger does not have SVX-II hits, therefore the trigger rate must be lowered so that track triggers do not dominate the trigger cross section. At level 1 two tracks with transverse momentum greater than $2.0 \mathrm{GeV} / \mathrm{c}$ will be required of opposite sign. A cut on the $\phi$ angle between the tracks may also be required. The trigger rate is estimated to be $16 \mathrm{kHz}$ for the luminosity expected in Run-II. The SVT will then require that both tracks have an impact parameter $\geq 100 \mu \mathrm{m}$ with respect to the beam and this should reject enough events to drop the rate for scanning to level 3 to $20 \mathrm{~Hz}$.

Unfortunately, $B^{0} \rightarrow \pi^{+} \pi^{-}$has sources of physics background beyond the background from QCD tracks. The decays $B \rightarrow K \pi, B, \rightarrow K K$, and $B_{s} \rightarrow K \pi$ will all put events in the mass region of the $B$ meson. Figure 11 shows the effect of this physics background. For the purpose of this plot there is no combinatorial background or particle ID assumed. The branching fraction of the $B \rightarrow \pi \pi$ decay mode is assumed to be equal to the branching fraction of $B \rightarrow K \pi$. The two $B$, branching fractions were also assumed to be equal to the $B^{ \pm}$counterparts but are scaled by the production fraction relative to $B$ mesons. From this plot it is clear that additional particle ID would be helpful for measuring $\sin 2 \alpha$. According to Monte Carlo studies, with $S / B k g \simeq 1.0$ it may be possible to expect $\delta \sin 2 \alpha \simeq 10 \%$.

\section{Conclusion}

The Collider Detector Facility has made significant contributions to the study of bottom quarks over the last 5 years. This is due to the high 
$B$ meson cross section but also because CDF has had a silicon detector that enables the detection of $B$ mesons by their decay length. Using this detector CDF has obtained competitive measurements on the production of $B$ mesons and quarkonia states, the direct observation of the $B$ lifetime, new particle and rare decay searches, and in the area of $B$ meson mixing and $B$ flavor tagging.

Because of the even higher luminosity for the next running period, significant upgrades are planned at CDF to take advantage of the silicon resolution in the trigger. This will allow CDF to take advantage of the hadronic decays of the $B$ meson in searches for CP violating effects. Further advances in trigger and data aquisition should only increase the trigger cross section allowing CDF and hadron machines in general to continue to contribute significantly to the rich field of Beauty physics.

\section{References}

[1] F. Abe et al., Nucl. Instrum. Methods Phys. Res., Sect. A 271, 387 (1988).

[2] In $\mathrm{CDF}, \varphi$ is the azimuthal angle, $\theta$ is the polar angle measured from the proton direction, and the $z$-axis is the beam axis.

[3] F. Abe et al., Phys. Rev. D52, 4784 (1995).

[4] F. Abe, et al., Phys. Lett. 69:3704-3708 (1992).

[5] P. Cho and A. Lebovich, Phys. Rev. D53:150 (1996).

[6] P. Dawson, et al., Nucl. Phys. B327, 49 (1988); M. Mangano, et al., Nucl. Phys. B373, 295 (1992).

[7] A. Martin, R. Roberts, and J. Stirling, Phys. Rev. D 47, 867 (1993).

[8] C. Peterson, et al., Phys. Rev. D 27, 105 (1983); J. Chrin, Z. Phys. C 36, 163 (1987).

[9] Albajar, et al., Phys. Lett. B 273, 540 (1991).

[10] R. Akers, et al., Z. Phys. C69, 195-214 (1996); P. Abreu, et al., Phys. Lett. B374, 351-361 (1996); D. Buskulic, et al., Phys. Lett. B357, 685 (1995). 
[11] M. Masetti and F. Sartogo, Phys. Lett. B357, 659-665, (1995); M. Lusignoli, M. Masetti, and S. Petraca, Phys. Lett. B266, 142-146 (1991).

[12] C.T.H. Davies, K. Hornbostel, G.P. Lepage, A.J. Lidsey, J. Shigemitsu, and J. Sloan, HEPLAT-9602020, (1996); E. Eichten and C. Quigg, Phys. Rev. D 49, 5845 (1995).

[13] M. Beneke and G. Buchalla, Phys. Rev. D 51, 4991 (1996); I.I. Bigi, Phys. Lett. B371, 105-110 (1996).

[14] R. Hawkings, This conference, June, 1996.

[15] M. Gronau and J.L. Rosner, Phys. Rev. D49, 254 (1994).

[16] The CDF collaboration Technical Design Report, 1996.

[17] F.Abe et al., Phys.Rev.Lett.73, 225-231,(1994).

[18] M. Ciuchini et. al., Z. Phys. C68, 239, 1995. 


\begin{tabular}{|c|c|c|}
\hline \multicolumn{3}{|c|}{ Comparison of $B$ Hadron Lifetimes } \\
all times in $p s$ \\
\hline$B$ Hadron & LEP (combined) & CDF \\
\hline$B^{0}$ & $1.52 \pm 0.05$ & $1.56 \pm 0.07$ \\
\hline$B^{+}$ & $1.63 \pm 0.06$ & $1.66 \pm 0.07$ \\
\hline$B_{s}^{0}$ & $1.60 \pm 0.10$ & $1.37 \pm 0.14$ \\
\hline$\Lambda_{b}$ & $1.21 \pm 0.07$ & $1.32 \pm 0.17$ \\
\hline Inclusive $B$ & $1.539 \pm 0.018$ & $1.524_{-0.030}^{+0.038}$ \\
\hline$\tau\left(B^{+}\right) / \tau\left(B^{0}\right)$ & $1.08 \pm 0.05$ & $1.05 \pm 0.06$ \\
\hline
\end{tabular}

Table 1: The table shows the CDF averaged measurements of the lifetimes of several $B$ hadrons. Also shown for comparison are the averaged results from LEP that were shown at this conference.

\begin{tabular}{|l|c|c|}
\hline Detector & CTC & COT \\
\hline Gas & Ar-Et $(50: 50)$ & Ar-Et-CF 4 (50:35:15) \\
Max. Drift Dist. & $3.6 \mathrm{~cm}$ & $0.88 \mathrm{~cm}$ \\
Max. Drift Time & $706 \mathrm{~ns}$ & $100 \mathrm{~ns}$ \\
Lorentz Angle & $45^{\circ}$ & $35^{\circ}$ \\
Drift Feild & $1.35 \mathrm{kV} / \mathrm{cm}$ & $2.6 \mathrm{kV} / \mathrm{cm}$ \\
Total Sense Wires & 6156 & 30,240 \\
Total Wires & 36,504 & 63,000 \\
Rad. Lengths & $1.7 \%$ & $1.3 \%$ \\
L1 Trig. $\Delta P_{T} / P_{T}^{2}$ & $.03 / \mathrm{GeV}$ & $.014 / \mathrm{GeV}$ \\
\hline
\end{tabular}

Table 2: Comparison of main design specifications between the Central tracking chamber (CTC) from 1986-1996 and the proposed Central Opencell Tracker (COT) for Run-II at 132 ns operation. 


\begin{tabular}{|l|c|c|}
\hline Detector & SVX' & SVX-II \\
\hline Coordinates & $r-\phi$ & $r-\phi, r-z$ \\
No. of Barrels & 2 & 3 \\
Layers per Barrel & 4 & 5 \\
Ladder length & $25.5 \mathrm{~cm}$ & $29.0 \mathrm{~cm}$ \\
Total Length & $51 \mathrm{~cm}$ & $87 \mathrm{~cm}$ \\
Inner Radius & $3.0 \mathrm{~cm}$ & $2.55 \mathrm{~cm}$ \\
Outer Radius & $7.8 \mathrm{~cm}$ & $10.6 \mathrm{~cm}$ \\
$r-\phi$ Strip Pitch & $60 ; 60 ; 60 ; 55 \mu \mathrm{m}$ & $60 ; 62 ; 60 ;$ \\
& & $60 ; 65 \mu \mathrm{m}$ \\
$r-z$ Strip Pitch & $\mathrm{N} / \mathrm{A}$ & $141 ; 125 ;$ \\
& & $60 ; 141 ; 65 \mu \mathrm{m}$ \\
No. of Channels & 46,080 & 405,504 \\
\hline
\end{tabular}

Table 3: Comparison of the current SVX' with the specifications of the next generation silicon detector for Run-II (the SVX-II detector).

\begin{tabular}{|l|c|c|c|}
\hline Tagging Method & $\begin{array}{c}\epsilon D^{2}(\%) \\
\text { (measured) }\end{array}$ & $\begin{array}{c}\epsilon D^{2}(\%) \\
\text { (expected) }\end{array}$ & Relevant Run II Upgrade \\
\hline \hline Jet Charge & $1.3 \pm 0.3$ & 3.0 & SVX II. Fwd. trk \\
Central Muon & $0.7 \pm 0.2$ & 0.7 & Completed muon coverage \\
Electron & & 1.0 & Plug cal. Fwd. Trk. \\
Same-side pion (SS) & $1.5 \pm 0.9$ & 2.0 & SVX II Fwd. Trk. \\
\hline Opposite-side Kaon & & 3.0 & Time of Flight \\
\hline
\end{tabular}

Table 4: Table showing the tagging asymmetry figure of merit $\left(\epsilon D^{2}\right)$ for various methods of tagging the $B$ meson flavor at the time of production as measured in Run-I. The projected values for Run-II are also shown and the upgrades responsible for the expected change. 


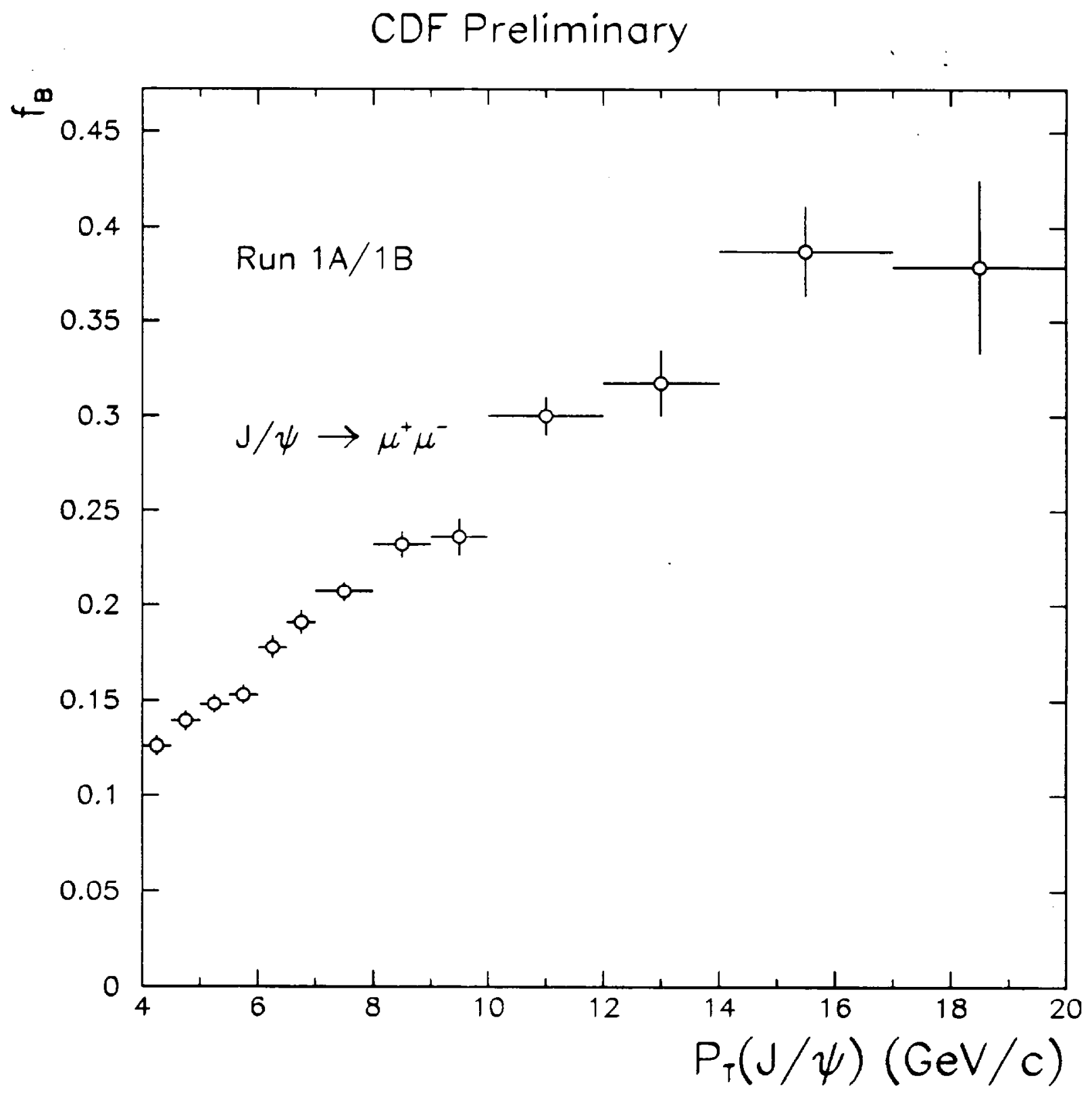

Figure 1: The fraction of $J / \psi$ 's that originate from $B$ meson decays as a function of the transverse momentum. This value $\left(f_{B}\right)$ is determined by fitting the $c \tau^{*}$ distribution of the signal events to both prompt and longlived components in the SVX. 

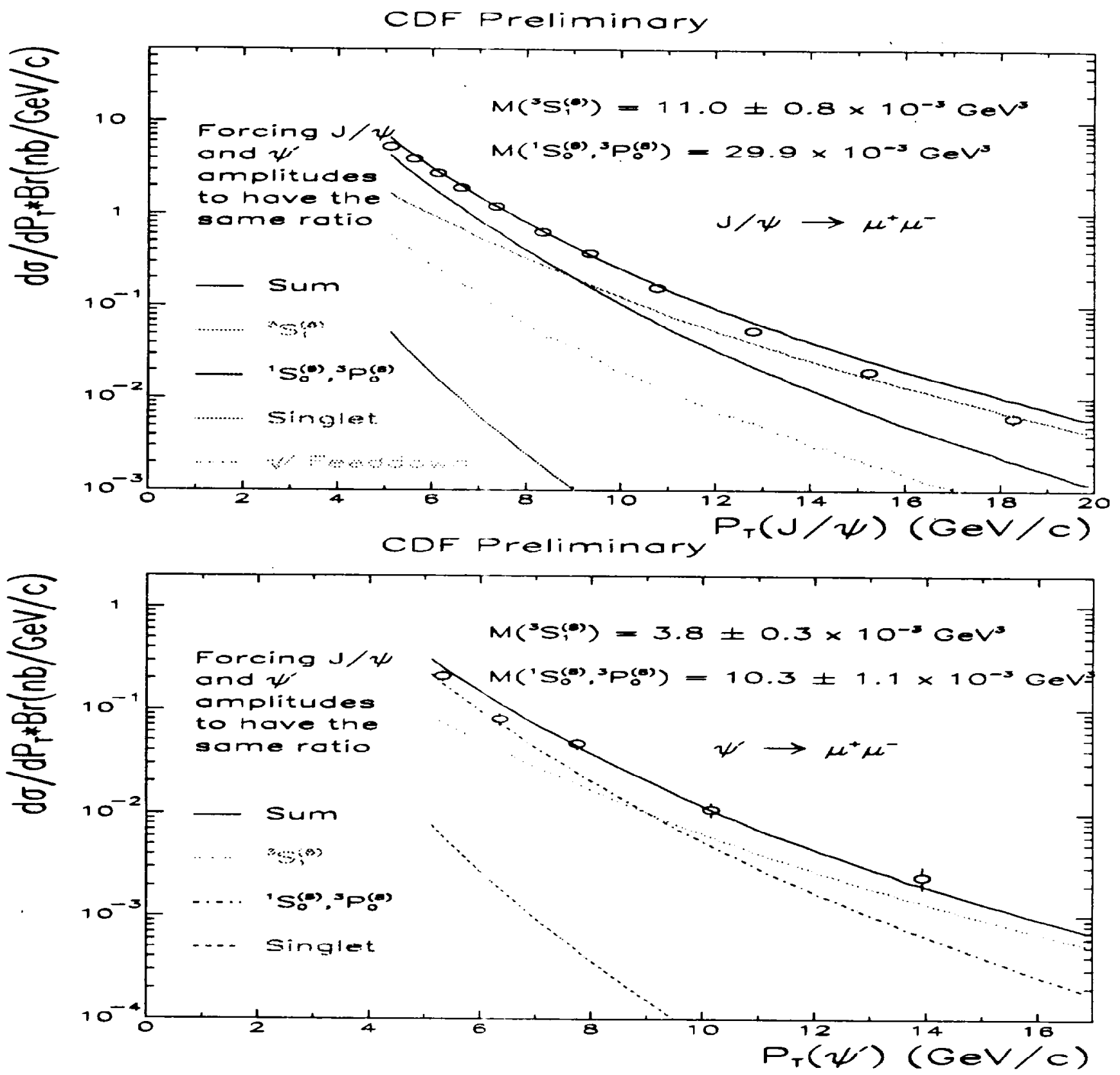

Figure 2: Top: The cross section from prompt production in $p-\bar{p}$ collisions of the $J / \psi$. Bottom: The $\psi(2 S)$ prompt cross section. Both plots use the decay of the charmonium state to muons pairs in the analysis. 


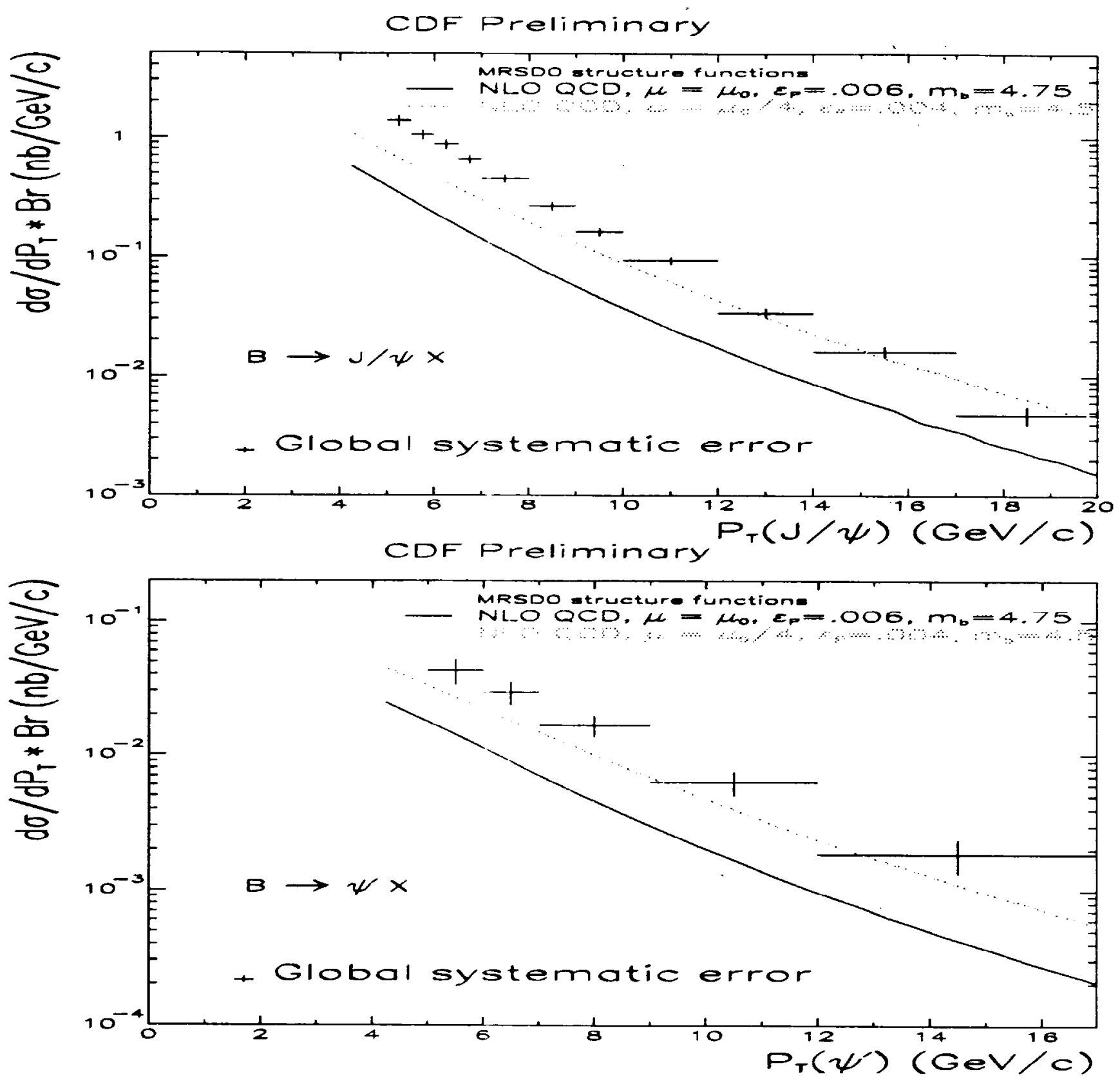

Figure 3: Top: The $J / \psi$ cross section from $b$ quark production in $p-\bar{p}$ collisions. Bottom: The $\psi(2 S)$ cross section from $b$ quark production. In both plots the solid line 


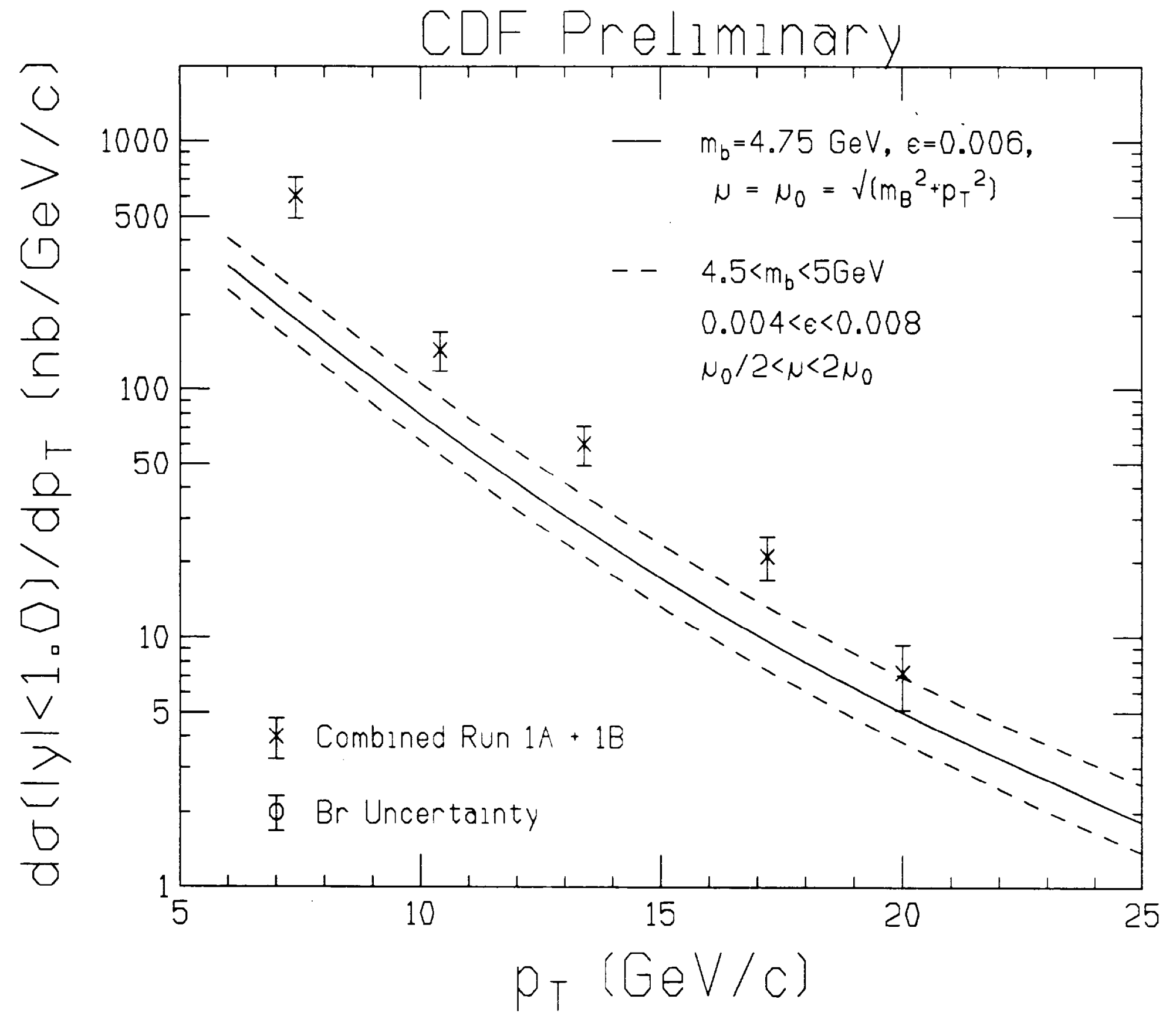

Figure 4: The differential cross section of the $B$ meson from exclusive decays to $J / \psi+K$ final states. The data combines the first and second parts of Run-I and a branching ratio uncertainty that would cause all points to shift up or down equally is not included with the points. 


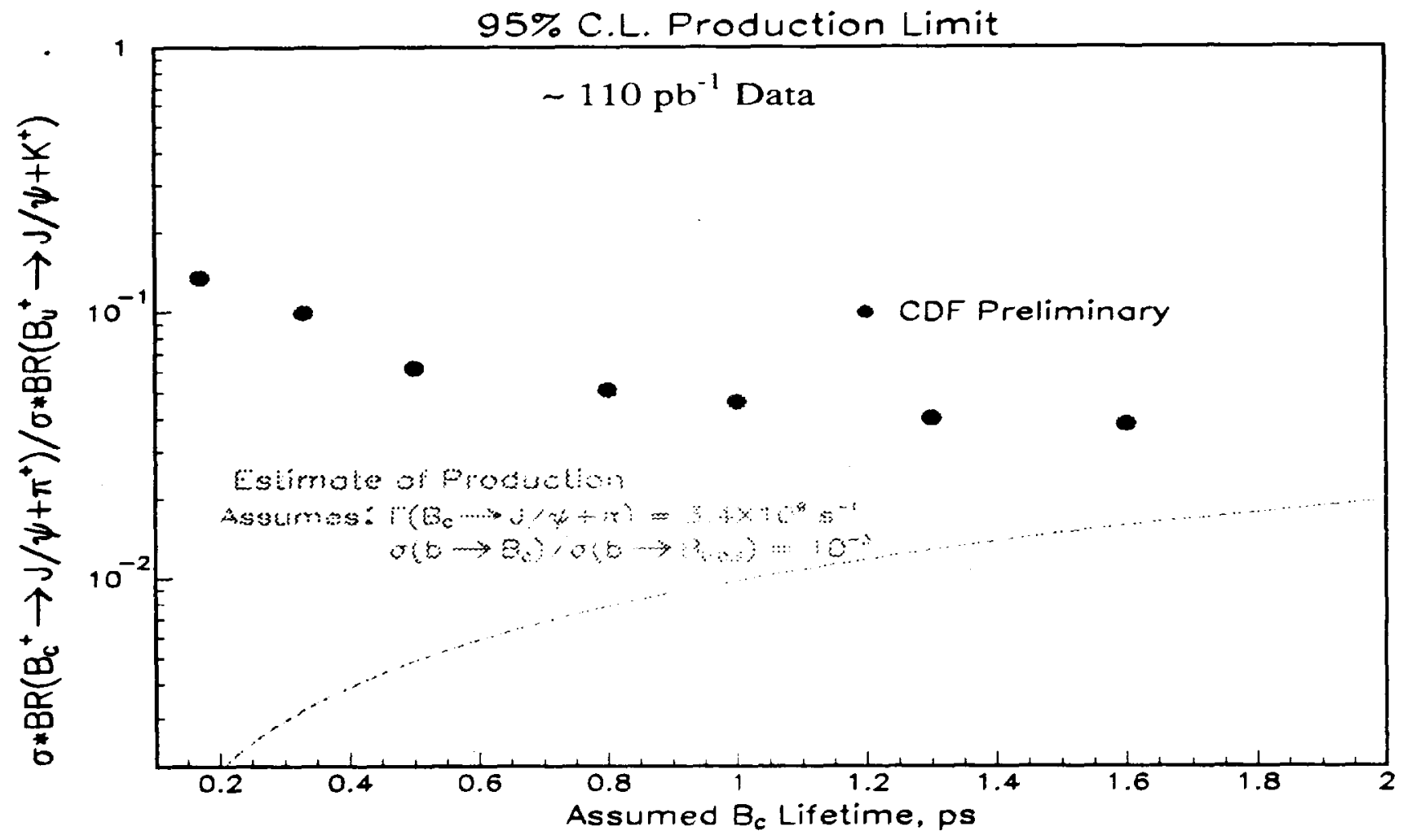

Figure 5: Shown is $\sigma \cdot \mathrm{BR}$ for $B_{c}^{+} \rightarrow J / \psi+\pi^{+}$relative to the observed decay $B_{u}^{+} \rightarrow J / \psi+K^{+}$. The dashed curve is the production one would receive if the $B_{c}$ meson is produced $10^{3}$ times less often than normal $B$ production and given the partial rate $\Gamma=3.4 \times 10^{9} \mathrm{~s}^{-1}$. This rate is based on the partial rate of the decay $B \rightarrow D^{*}+\pi$ which has similar phase space and form factors. 


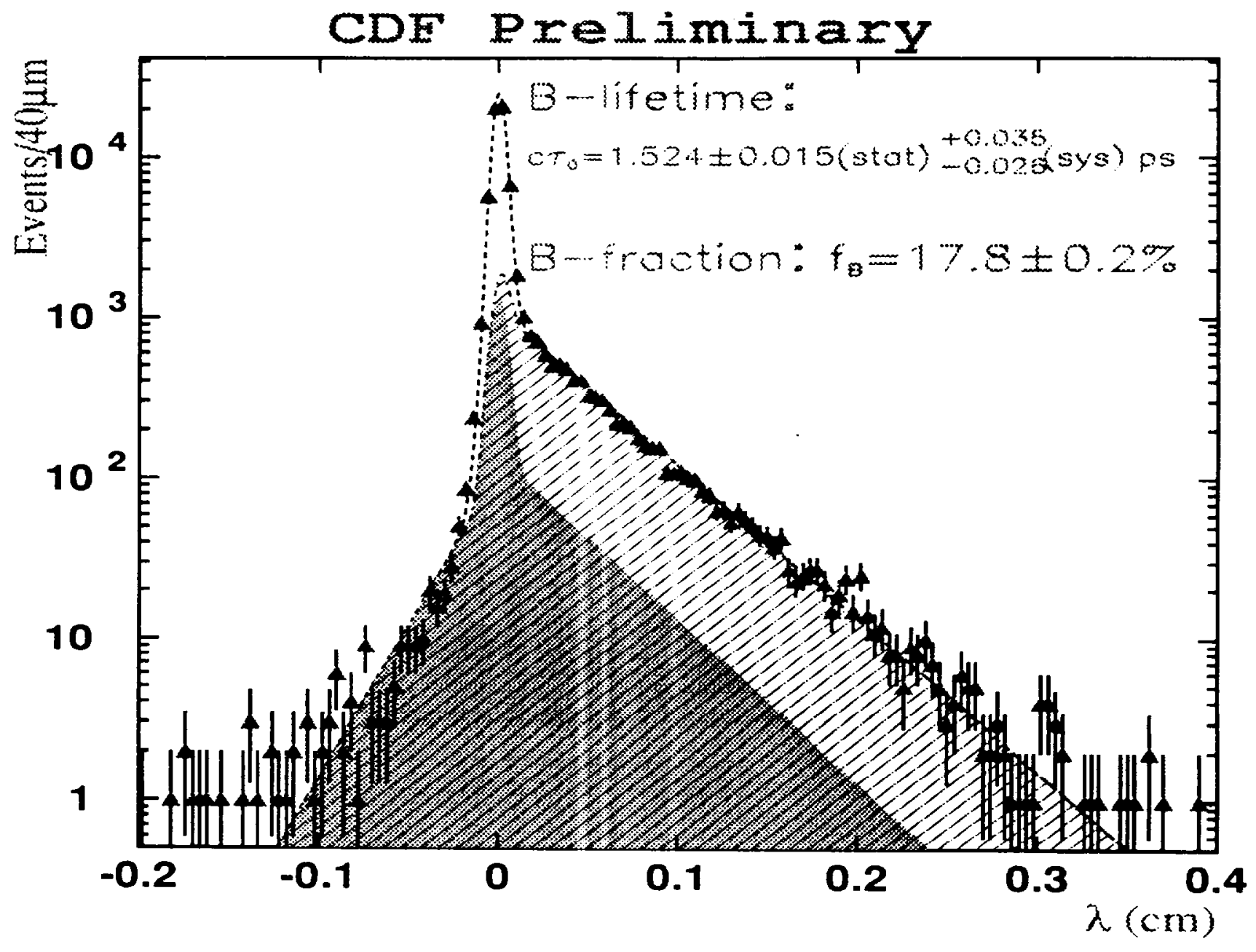

Figure 6: The figure shows the psuedo-c $\tau$ distribution for $J / \psi$ 's. Fitting this plot determines the integrated lifetime of the long-lived $B$ component and measures the fraction of bottom hadrons that produce $J / \psi$ 's in the data. 


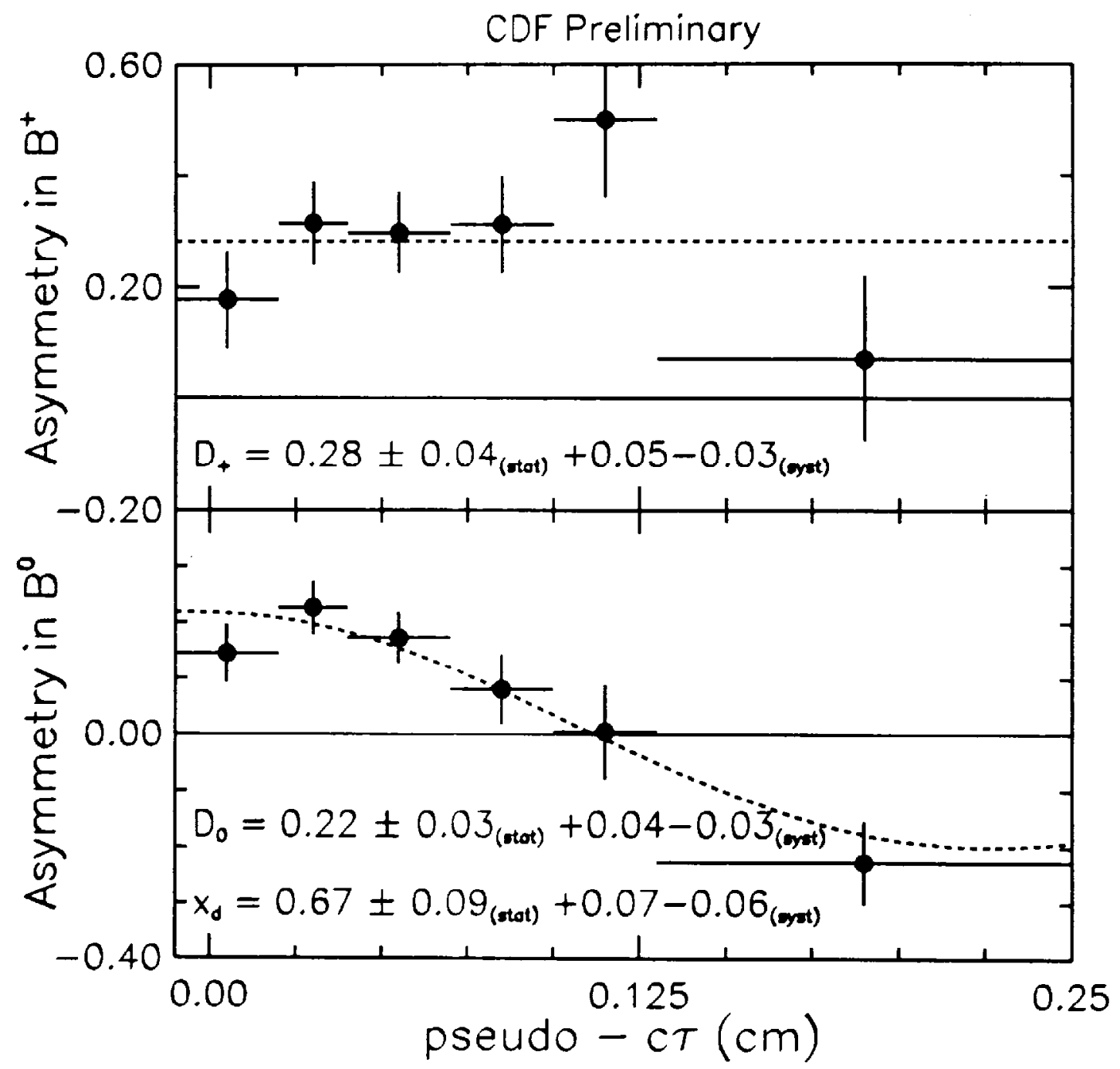

Figure 7: Time dependent dilutions for neutral $B\left(D_{0}\right)$ and for charged $B$ $\left(D_{+}\right)$, in the case where a cut on impact parameter significance of the $B$ meson daughter tracks has been applied. The property of $B^{0}-\bar{B}^{0}$ mixing is evident in the lower plot. 


\section{Dataflow of CDF "Deadtimeless" Trigger and DAQ}

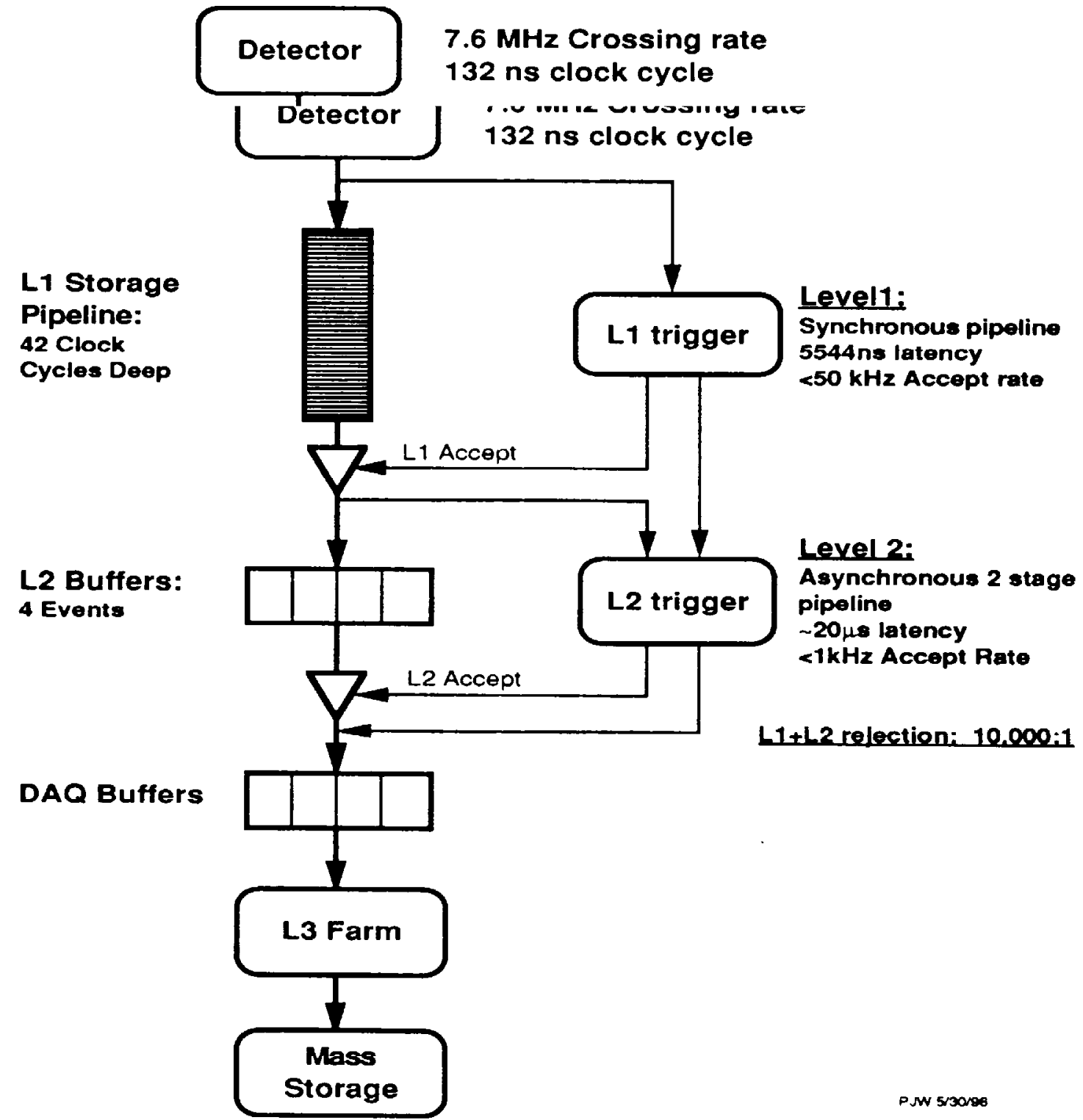

Figure 8: Shown is the data flow for the proposed CDF "deadtimeless" data system. The system is deadtimeless in the sense that data can continue to enter the system while previous events are in processing as long as there are available buffers. 


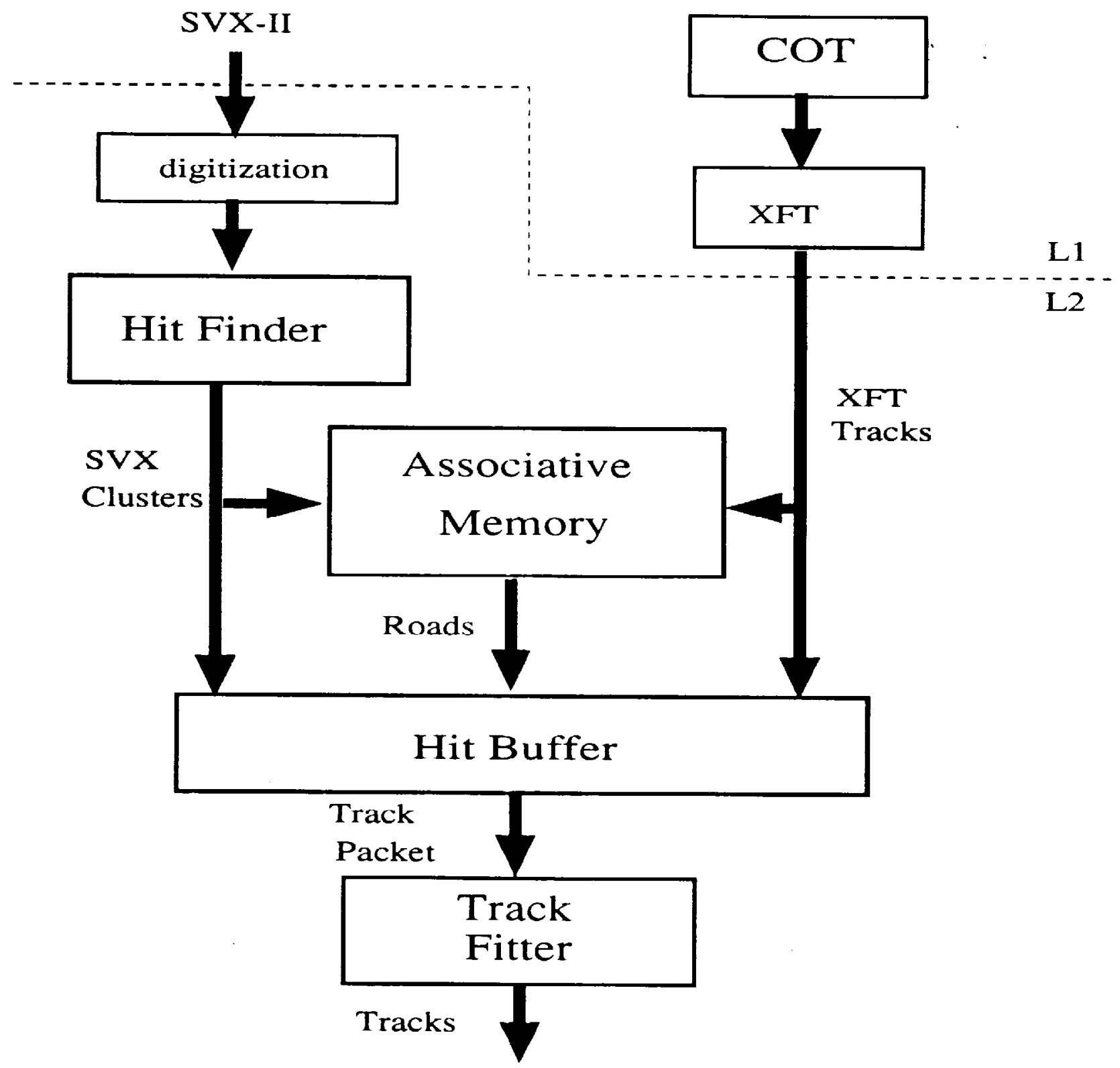

\section{L2 Decision}

Figure 9: Shown is the data path taken by events in the Silicon Vertex Trigger. This trigger searches for silicon detector tracks with large impact parameter with respect to the beam spot and matches them with tracks from the central tracker. 


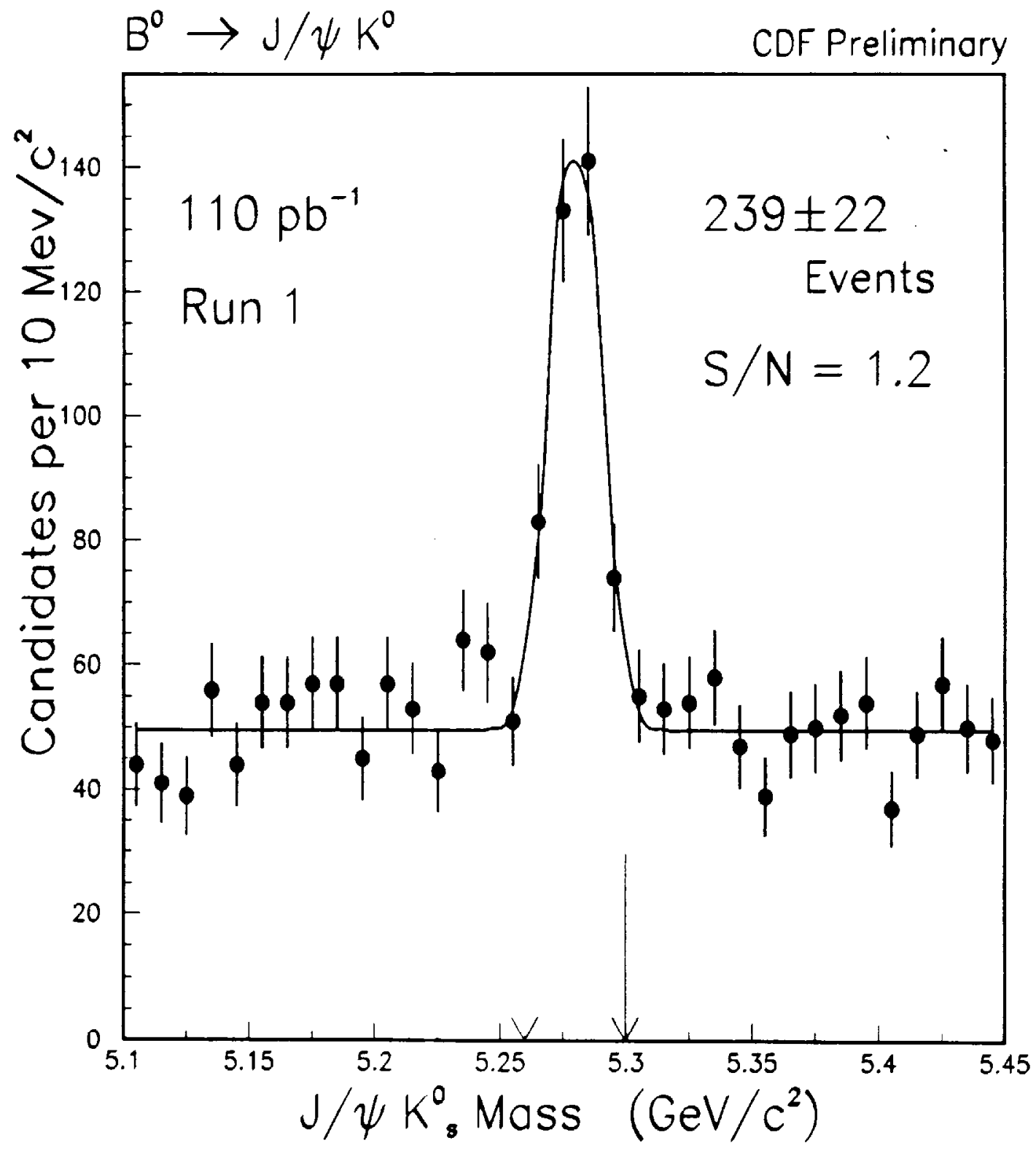

Figure 10: This plot shows the total number of $B$ mesons collected in $110 \mathrm{pb}^{-1}$ of data for the decay to $J / \psi+K_{\mathrm{s}}^{0}$. It is from this starting point from which our arguments of CP violation reach in Run-II proceed. 


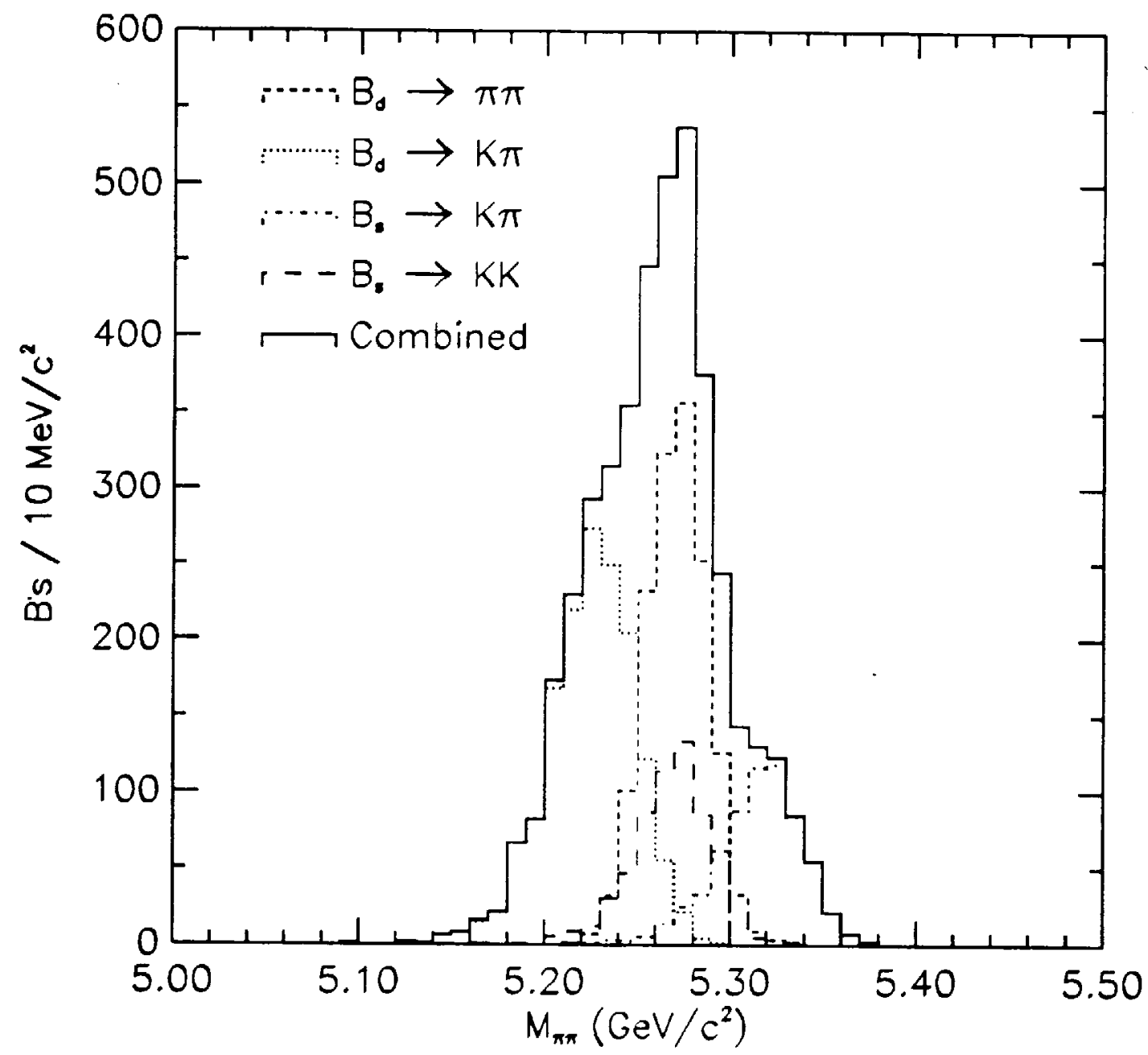

Figure 11: This plot illustrates one of the challenges involved in measuring the CP angle $\alpha$ in the unitarity triangle. The decays $B \rightarrow \pi \pi, B \rightarrow$ $K \pi, B_{s} \rightarrow K K$, and $B, \rightarrow K \pi$ are shown supperimposed as they would appear with no particle ID to discriminate between kaons and pions. Equal branching fractions are assumed to the $K \pi$ and $\pi \pi$ modes are assumed and the relative production of $B$ to $B$, of $12 \%$ is included. No background is shown in this plot. The mass resolution is assumed to be $20 \mathrm{MeV} / \mathrm{c}^{2}$ in this figure. 